\title{
Routine surveillance of patients post Fontan palliation: lessons learnt from cardiac catheterisation
}

\author{
Maria Victoria Ordonez ${ }^{1,2^{*}}$ (D, Giovanni Biglino ${ }^{1,3}$ and Radwa Bedair ${ }^{2}$
}

\begin{abstract}
Background: There is no consensus on the clinical utility of 'routine' diagnostic cardiac catheterisation in patients with Fontan palliation in the absence of symptoms or haemodynamic lesions.

Objective: We sought to evaluate whether diagnostic cardiac catheterisation for a variety of indications led to a change in the clinical management of patients with a Fontan circulation.

Methods: All adult patients ( $\geq 16$ years) with Fontan palliation undergoing diagnostic cardiac catheterisation at our institution from 2016 to 2019 were included retrospectively. Patients undergoing electrophysiological studies were excluded as haemodynamic measurements were not taken. Routine cardiac catheterisation at our institution is considered in adult patients who have not had a diagnostic cardiac catheter for more than 5 years.

Results: Thirty-eight patients, mean age $27 \pm 7$ years, 60\% NYHA I, 31\% NYHA II, 8\% NYHA III, at mean duration post Fontan of $20 \pm 6$ years, lateral tunnel (LT) $n=20$, extracardiac (EC) $n=14$ and atriopulmonary (AP) $n=4$, underwent 41 diagnostic cardiac catheterisation procedures. Indication for cardiac catheterisation was as follows: haemodynamic lesion identified on cross-sectional imaging in 12; routine catheterisation in 9; cyanosis in 8; dyspnoea in 8; significant liver stiffness on ultrasound hepatic elastography in 2; and arrhythmia in 2. Of the 9 patients undergoing routine diagnostic catheterisation, 3 had not had any diagnostic catheterisation since their Fontan completion and, in the remaining six, the mean time lapsed since the last diagnostic catheter was $8 \pm 3$ years. The diagnostic catheterisation led to a recommended change in clinical management on 24 occasions (59\%): catheter intervention in 17 (40\%); surgery in 4 (10\%); medication change in $3(17 \%)$; and transplant referral in $2(5 \%)$. The clinical indications that led to changes in clinical management were: cyanosis (8/8), dyspnoea (7/8), haemodynamic lesions on cross-sectional imaging (8/11) and arrhythmia (1/2). None of the 9 patients listed for routine diagnostic catheterisation or as a result of findings on ultrasound hepatic elastography had a recommended change in clinical management.

Conclusion: Diagnostic cardiac catheterisation frequently leads to changes in the clinical management of patients with Fontan palliation presenting with dyspnoea, cyanosis, and for further evaluation of potential haemodynamic lesions identified on cross-sectional imaging. Routine cardiac catheterisation in the absence of the above indications had limited impact on clinical management in our cohort.
\end{abstract}

Keywords: Fontan palliation, Routine diagnostic catheterisation, Clinical indicated catheterisation, Long term follow up

*Correspondence: victoria.ordonez@uhbw.nhs.uk

'Bristol Medical School, University of Bristol, Bristol, UK

${ }^{2}$ Adult Congenital Heart Disease Service, University Hospitals Bristol, NHS

Foundation Trust, Bristol, UK

Full list of author information is available at the end of the article

(c) The Author(s). 2021 Open Access This article is licensed under a Creative Commons Attribution 4.0 International License, which permits use, sharing, adaptation, distribution and reproduction in any medium or format, as long as you give appropriate credit to the original author(s) and the source, provide a link to the Creative Commons licence, and indicate if changes were made. The images or other third party material in this article are included in the article's Creative Commons licence, unless indicated otherwise in a credit line to the material. If material is not included in the article's Creative Commons licence and your intended use is not permitted by statutory regulation or exceeds the permitted use, you will need to obtain permission directly from the copyright holder. To view a copy of this licence, visit http://creativecommons.org/licenses/by/4.0/. The Creative Commons Public Domain Dedication waiver (http://creativecommons.org/publicdomain/zero/1.0/) applies to the data made available in this article, unless otherwise stated in a credit line to the data. 


\section{Introduction}

Most Fontan patients reach adulthood [1-3]; however, a standardised long-term follow-up strategy has not been agreed, partly reflecting the non-standardised management of these challenging patients across institutions. Fontan patients can develop several complications such as atrial arrhythmias, heart failure, protein losing enteropathy, plastic bronchitis, progressive cyanosis due to veno-venous collateral formation, and Fontan-associated liver disease (FALD), which are the mainstay of Fontan morbidity and mortality [3, 4] (Table 1 ). The risk and severity of these complications increases over time and with advancing age, and early identification and treatment of any haemodynamic drivers for the development of these complications is essential [3]. Surveillance protocols differ substantially across centres and the unification of these patients' follow-up remains challenging. Previous studies suggested that these patients present a bimodal pattern, with a cumulative risk of death from thromboembolism increasing during the first 3 years after Fontan palliation and again 15 years after surgery $[4,5]$, reiterating the importance of surveillance. Recent findings indicate that the use of cardiac MRI for postFontan surveillance follows this bimodal trend [1], with significantly greater odds of a change in management following clinically indicated MRI after Fontan as compared to screening MRI. We here present complementary observations based on cardiac catheterisation.

\section{Methods}

We retrospectively analysed $n=38$ adult Fontan patients who underwent diagnostic cardiac catheterisation between 2016 and 2019, mean follow up post-Fontan palliation: $20 \pm 6$ years, type of Fontan: LT $n=20$, EC $n=14$ and AP $n=4$. We compared patients who underwent routine diagnostic cardiac catheterization $(n=9)$ versus clinically-indicated diagnostic catheterisation $(n=29)$.
Routine catheterisation at our institution is considered in adult patients who have not had a diagnostic cardiac catheter for more than 5 years. Clinical indications for diagnostic catheterisation are displayed in Fig. 1 and included cross-sectional imaging study, arrhythmias, cyanosis, dyspnoea and cirrhotic liver.

\section{Results}

Diagnostic catheterisation led to a recommended change in clinical management in 24 occasions (59\%): catheter intervention in 17 (40\%); surgery in 4 (10\%); medication change in 3 (17\%); and transplant referral in 2 (5\%) (Fig. 1 ). None of the 9 patients listed for routine diagnostic catheterisation had a recommended change in clinical management following the procedure.

\section{Discussion}

Similar to MRI observations [1], our findings show limited clinical utility and impact on changing management from routine testing in Fontan patients. However, no definitive conclusion could be drawn based on the small sample. Additionally, the reassurance that a favourable result from a diagnostic cardiac catheterisation study brings to the patient and medical team has not been explored, and may be important. Monitoring for complications in Fontan patients encompasses liver surveillance, single ventricle function assessment, and arrhythmias follow-up. Based on AHA guidelines [3], Fontan patients should be evaluated annually with either echocardiography or cardiac MRI (class I level of evidence C) and cardiac catheterization should be performed in adults before initial Fontan surgery or revision of a prior Fontan connection to assess suitability of pre-intervention hemodynamics for Fontan physiology (class I level of evidence $\mathrm{C}$ ), in the context of a new onset or worsening atrial tachyarrhythmias (class I level of evidence C), or when the patient is symptomatic and non-invasive testing is insufficient to guide therapy (class IIa level of evidence C), as well as

Table 1 Fontan complications and imaging modalities for assessing and following Fontan patients

\begin{tabular}{|c|c|c|c|c|c|}
\hline Fontan Complications & Echo & CMR & CT & Cath & Additional imaging modalities \\
\hline Conduit Obstruction & $\checkmark \checkmark \checkmark$ & $\checkmark \checkmark \checkmark$ & $\checkmark \checkmark \checkmark$ & $\checkmark \checkmark \checkmark$ & \\
\hline Pulmonary branches stenosis & & $\checkmark \checkmark \checkmark$ & $\checkmark \checkmark \checkmark$ & $\checkmark \checkmark \checkmark$ & \\
\hline Veno-venous collaterals & & $\checkmark \checkmark \checkmark$ & & $\checkmark \checkmark \checkmark$ & \\
\hline Arrhythmias & $\checkmark \checkmark$ & $\checkmark \checkmark \checkmark$ & & $\checkmark \checkmark \checkmark$ & \\
\hline$A \bigvee$ valve regurgitation & $\checkmark \checkmark$ & $\checkmark \checkmark \checkmark$ & & & \\
\hline Aortic regurgitation & $\checkmark \checkmark$ & $\checkmark \checkmark \checkmark$ & & & \\
\hline Decreased preload & $\checkmark \checkmark$ & $\checkmark \checkmark$ & & $\checkmark \checkmark \checkmark$ & \\
\hline Systolic dysfunction & $\checkmark \checkmark$ & $\checkmark \checkmark \checkmark$ & & & \\
\hline Increased afterload & $\checkmark \checkmark \checkmark$ & $\checkmark \checkmark \checkmark$ & & $\checkmark \checkmark \checkmark$ & \\
\hline Liver abnormalities & $\checkmark \checkmark \checkmark$ & $\checkmark \checkmark \checkmark$ & $\checkmark \checkmark \checkmark$ & & Liver Ultrasound Fibroscan \\
\hline Protein losing enteropathy & $\checkmark \checkmark \checkmark$ & $\checkmark \checkmark \checkmark$ & & $\checkmark \checkmark \checkmark$ & \\
\hline Plastic bronchitis & & $\checkmark \checkmark \checkmark$ & & $\checkmark \checkmark \checkmark$ & Bronchoscopy \\
\hline
\end{tabular}




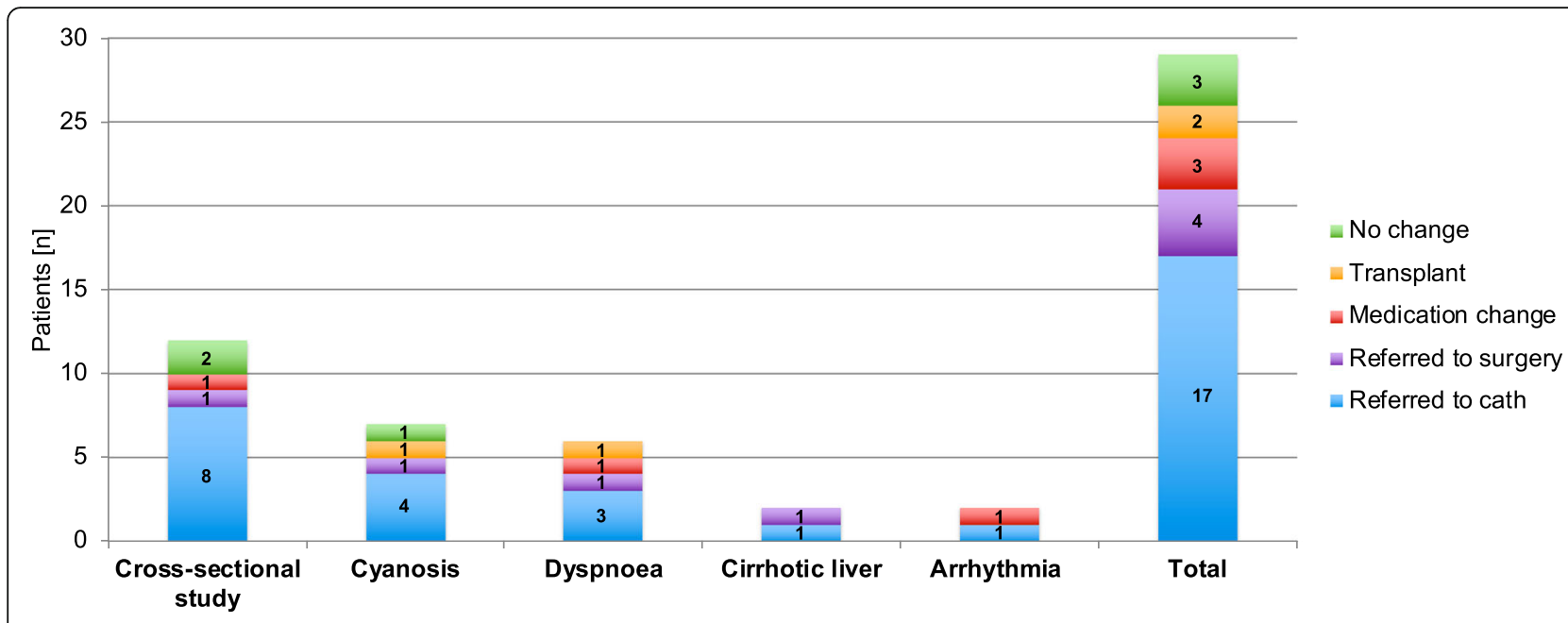

Fig. 1 Clinical indications and changes in clinical management post diagnostic catheterisation

before cardiac transplantation. Evidence-based treatment strategies and preventive management schema are limited, with a need for more widespread consensus as to how best serially monitoring and managing these patients [6] in order to establish a safe protocol.

\section{Conclusion}

Clinically-indicated diagnostic catheterisation frequently leads to changes in the clinical management with Fontan palliation with limited impact on routine diagnostic catheterisation in our cohort. Nonetheless, given the fragile and delicate balance of the univentricular physiology, diagnostic catheterisation might play an essential role as part of the long term follow up in Fontan patients by identifying hemodynamic abnormalities in the context of subtle or even in the absence of clinical manifestations. Therefore, further analysis is warranted.

\section{Acknowledgments}

British Heart Foundation (BHF), Biomedical Research Center (BRC).

\section{Authors' contributions}

Dr. Maria Victoria Ordonez collected and organized the data, analysed the results with the clinical data, was involved in the writing process. Moreover, the study was presented as a poster at the CSI conference in Frankfurt, June 2019 by Dr. Maria Victoria Ordonez. Dr. Giovanni Biglino did the statistical analysis and was involved in the writing process. Dr. Radwa Bedair set the goals, coordinated the study and was involved in the writing process as an Interventional Cardiologist. All the authors have read and approved the final manuscript.

\section{Funding}

None.

Availability of data and materials

RedCup dataset, Bristol Heart Institute and Bristol University.

\section{Declarations}

\section{Ethics approval and consent to participate}

The present retrospective analysis was approved by the audit board at University Hospital of Bristol.
Consent for publication

Not applicable.

\section{Competing interests}

None to declare.

\section{Author details}

${ }^{1}$ Bristol Medical School, University of Bristol, Bristol, UK. ${ }^{2}$ Adult Congenital Heart Disease Service, University Hospitals Bristol, NHS Foundation Trust, Bristol, UK. ${ }^{3}$ National Heart and Lung Institute, Imperial College London, London, UK.

Received: 18 May 2020 Accepted: 21 February 2021

Published online: 24 March 2021

\section{References}

1. Zaki NC, Kelleman MS, James Parks W, Slesnick TC, McConnell ME, Oster ME. The utility of cardiac magnetic resonance imaging in post-Fontan surveillance. Congenit Heart Dis. 2019;14(2):140-6.

2. Stout KK, Daniels CJ, Aboulhosn JA, Bozkurt B, Broberg CS, Colman JM, et al. 2018 AHA/ACC guideline for the management of adults with congenital heart disease: a report of the American College of Cardiology/American Heart Association task force on clinical practice guidelines. Circulation. 2019; 139(14):e698-800.

3. Inglessis I, Landzberg MJ. Interventional catheterization in adult congenital heart disease. Circulation. 2007:115(12):1622-33.

4. Khairy P, Fernandes SM, Mayer JE, Triedman JK, Walsh EP, Lock JE, Landzberg MJ. Long-term survival, modes of death, and predictors of mortality in patients with Fontan surgery. Circulation. 2008;117(1):85.

5. d'Udekem Y, lyengar AJ, Galati JC, Forsdick V, Weintraub RG, Wheaton GR, et al. Redefining expectations of long-term survival after the Fontan procedure: twenty-five years of follow-up from the entire population of Australia and New Zealand. Circulation. 2014;130(11_suppl_1):S32-8.

6. Rychik J, Atz AM, Celermajer DS, Deal BJ, Gatzoulis MA, Gewillig MH, Hsia TY, Hsu DT, Kovacs AH, McCrindle BW, Newburger JW. Evaluation and management of the child and adult with Fontan circulation: a scientific statement from the American Heart Association. Circulation. 2019;140(6): e234-84.

\section{Publisher's Note}

Springer Nature remains neutral with regard to jurisdictional claims in published maps and institutional affiliations. 